\title{
Paediatric crossword puzzle - 19 (Answers)
}

Manouri P. Senanayake ${ }^{1}$

Sri Lanka Journal of Child Health, 2013; 42(3): 174

\begin{tabular}{|c|c|c|c|c|c|c|c|c|c|c|c|c|c|}
\hline${ }^{1} \mathrm{~A}$ & $S$ & $D$ & & & & ${ }^{2} \mathrm{~T}$ & & & & & & ${ }^{3} S$ & $M$ \\
\hline$P$ & & & ${ }^{4} B$ & 0 & ${ }^{5} \mathrm{~T}$ & $U$ & $L$ & 1 & ${ }^{6} \mathrm{~N}$ & $U$ & ${ }^{7} \mathrm{M}$ & & 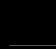 \\
\hline G & & & 1 & & ${ }^{8} I$ & $\mathrm{~N}$ & & & $E$ & & 0 & & ${ }^{9} \mathrm{~A}$ \\
\hline${ }^{10} \mathrm{~A}$ & $T$ & $Y$ & $P$ & I & $C$ & $A$ & $\mathrm{~L}$ & & $C$ & & $S$ & & $\mathrm{D}$ \\
\hline$R$ & & & $\mathrm{H}$ & & & & & & & & $A$ & & 0 \\
\hline & ${ }^{11} \mathrm{~A}$ & & $A$ & & ${ }^{12} \mathrm{P}$ & & & ${ }^{13} \mathrm{~N}$ & & & ${ }^{14} I$ & 0 & $\mathrm{~L}$ \\
\hline & $\mathrm{L}$ & & ${ }^{15} \mathrm{~S}$ & ${ }^{16} \mathrm{I}$ & G & ${ }^{17} \mathrm{M}$ & 0 & I & $D$ & & $C$ & & $E$ \\
\hline & $B$ & & ${ }^{18} \mid$ & $\mathrm{V}$ & I & $\bar{G}$ & & $\bar{L}$ & & ${ }^{19} \mathrm{~S}$ & I & $\mathrm{R}$ & $S$ \\
\hline${ }^{20} \mathrm{Z}$ & 1 & $\mathrm{~N}$ & $C$ & & $M$ & & & $\mathrm{~K}$ & & & $S$ & & $C$ \\
\hline & $\mathrm{N}$ & & & & & & ${ }^{21} \mathrm{C}$ & & ${ }^{2} \mathrm{~S}$ & $A$ & $M$ & & $\mathrm{E}$ \\
\hline & I & & ${ }^{23} \mathrm{~B}$ & & & & $\mathrm{H}$ & & & & & & $\mathrm{N}$ \\
\hline & ${ }^{24} \mathrm{~S}$ & $\mathrm{Y}$ & $P$ & ${ }^{5} \mathrm{H}$ & I & $\mathrm{L}$ & $I$ & $S$ & & ${ }^{26} \mathrm{C}$ & $\mathrm{R}$ & ${ }^{27} \mathrm{~F}$ & $T$ \\
\hline & $M$ & & & 1 & & & $\mathrm{~N}$ & & & $M$ & & $F$ & \\
\hline & & ${ }^{28} \mathrm{R}$ & 1 & $P$ & $E$ & & ${ }^{29} \mathrm{~A}$ & $D$ & $\mathrm{R}$ & $E$ & $\mathrm{~N}$ & $A$ & $\mathrm{~L}$ \\
\hline
\end{tabular}

${ }^{1}$ Professor in Paediatrics, Faculty of Medicine, University of Colombo 